Meta

Journal des traducteurs

Translators' Journal

\title{
Au tour de Babel ! Les défis multiples du multilinguisme
}

\section{Madeleine Stratford}

Volume 53, numéro 3, septembre 2008

URI : https://id.erudit.org/iderudit/019234ar

DOI : https://doi.org/10.7202/019234ar

Aller au sommaire du numéro

Éditeur(s)

Les Presses de l'Université de Montréal

ISSN

0026-0452 (imprimé)

1492-1421 (numérique)

Découvrir la revue

Citer cet article

Stratford, M. (2008). Au tour de Babel ! Les défis multiples du multilinguisme. Meta, 53(3), 457-470. https://doi.org/10.7202/019234ar

\section{Résumé de l'article}

Selon la conception traditionnelle de la traduction, les traducteurs ont pour tâche de camoufler Babel en corrigeant, en adaptant et en uniformisant les discours afin de faciliter la communication entre les peuples. Or, de plus en plus d'écrivains bilingues, voire polyglottes, célèbrent Babel en créant des textes où deux ou plusieurs langues cohabitent. Que se passe-t-il lorsque ces mondes entrent en collision et qu'un traducteur décide de relever les défis multiples que présente un texte multilingue ? La présente étude situe d'abord le multilinguisme littéraire à travers l'histoire, résume ensuite ses diverses manifestations et fonctions, pour enfin souligner l'importance chez les traducteurs et traductologues de développer de nouvelles stratégies pour faire rayonner Babel.
Ce document est protégé par la loi sur le droit d'auteur. L'utilisation des services d'Érudit (y compris la reproduction) est assujettie à sa politique d'utilisation que vous pouvez consulter en ligne.

https://apropos.erudit.org/fr/usagers/politique-dutilisation/ 


\title{
Au tour de Babel! Les défis multiples du multilinguisme
}

\author{
MADELEINE STRATFORD \\ Université Laval, Québec, Canada \\ brujileine@yahoo.com
}

\begin{abstract}
RÉSUMÉ
Selon la conception traditionnelle de la traduction, les traducteurs ont pour tâche de camoufler Babel en corrigeant, en adaptant et en uniformisant les discours afin de faciliter la communication entre les peuples. Or, de plus en plus d'écrivains bilingues, voire polyglottes, célèbrent Babel en créant des textes où deux ou plusieurs langues cohabitent. Que se passe-t-il lorsque ces mondes entrent en collision et qu'un traducteur décide de relever les défis multiples que présente un texte multilingue? La présente étude situe d'abord le multilinguisme littéraire à travers l'histoire, résume ensuite ses diverses manifestations et fonctions, pour enfin souligner l'importance chez les traducteurs et traductologues de développer de nouvelles stratégies pour faire rayonner Babel.
\end{abstract}

\begin{abstract}
According to the traditional view of translation, the translator's task consists in hiding Babel by correcting, adapting, and standardizing discourses to facilitate communication between different cultures. However, more and more bilingual or multilingual writers now celebrate Babel by producing texts where two or more languages come into contact. What happens when the two worlds collide, and when the translator decides to take on the challenge of translating a multilingual text? This paper first situates multilingualism in the history of literature, then summarizes its various manifestations and functions, and finally stresses the importance for translators and translation scholars to develop new strategies to let Babel shine.
\end{abstract}

\section{MOTS-CLÉS/KEYWORDS}

multilinguisme, littérature, hybridité, scepticisme linguistique, traductibilité

\section{Introduction}

Depuis des siècles, des traducteurs multilingues se consacrent à jeter des ponts entre les langues, les cultures et les peuples. Ils corrigent, adaptent, uniformisent les discours pour faciliter la communication. Pendant ce temps, d'autres polyglottes, plus téméraires peut-être, choisissent de célébrer Babel en créant des textes où deux ou plusieurs langues cohabitent. Que se passe-t-il lorsque les deux mondes entrent en collision et qu'un traducteur doit faire face à un texte multilingue? Dans cette étude, nous allons d'abord situer le phénomène du multilinguisme dans l'histoire de la littérature, pour résumer ensuite ses diverses manifestations et fonctions. Enfin, nous allons examiner dans quelle mesure il est possible ou non pour le traducteur de relever les défis que représentent les textes multilingues. 


\section{Le multilinguisme littéraire à travers les époques}

Dans l'histoire de la littérature, le multilinguisme est tantôt prisé, tantôt dédaigné: «on [le] verra [...] surgir comme une mode qui caractérise la littérature de toute une époque, pour disparaître ensuite complètement» (Elwert 1960: 416). Au Moyen-Âge, on répertorie notamment de nombreuses œuvres multilingues, tous genres confondus. À l'époque, la plupart des auteurs et des lecteurs sont polyglottes, une tendance qui perdure au moins jusqu'à la fin du XVII siècle (Mackey 1993: 43). Bien que les écrivains rédigent généralement leurs textes en latin classique, ils les parsèment fréquemment de vers ou de strophes dans la langue vernaculaire qui leur est propre (Forster 1970: 10; Steiner 1992: 197-198). Vers le XIX ${ }^{e}$ siècle, l'émergence du concept de «nation" amène les auteurs multilingues à choisir l'idiome qui marque leur appartenance à un pays. Même si une grande partie de la population continue de parler plusieurs langues dans la vie quotidienne, écrire devient un acte politiquement chargé de conséquences. Il n'est donc plus question pour les écrivains bilingues de produire des textes hybrides $^{1}$ (Mackey 1993 : 44). À la longue, par contre, le fait d'avoir octroyé une connotation "politique» aux langues nationales rend les écrivains de plus en plus méfiants envers leur médium: "Words become dirty because of their associations and the contexts in which they are used» (Forster 1970: 93).

Pendant la première moitié $\mathrm{du} \mathrm{xx}^{\mathrm{e}}$ siècle, les deux guerres mondiales obligent plusieurs écrivains à fuir leur pays d'origine pour en adopter un autre. Certains de ces exilés continuent d'écrire dans leur langue maternelle, tandis que d'autres préfèrent apprivoiser la langue de leur nouveau pays, "pour des raisons d'ordre pratique (contraintes éditoriales, nécessité de trouver un public) ou pour des motifs plus personnels (adaptation au nouvel environnement linguistique ou culturel)» (KleinLataud 1996: 212). Cependant, cet apprivoisement ne signifie pas nécessairement que leurs idiomes s'entremêlent dans un même texte. Quel que soit le degré de pénétration de l'élément étranger, le recours à une autre langue peut à l'occasion " [libérer] à la fois de la langue maternelle et du passé avec lequel celle-ci fait bloc» (Klein-Lataud 1996: 215). Néanmoins, un dédoublement linguistique est également susceptible de générer une remise en question de nature ontologique chez l'écrivain polyglotte, comme le constate Steiner: "In what language am I, suis-je, bin ich [...] ?» (Steiner 1992: 125). Certains écrivains multilingues sont incapables de s'exprimer, voire de penser, en une seule langue (Mackey 1993: 50). Dans ces cas, l'hétéroglossie d'un texte littéraire est notamment symptomatique d'un malaise profond de l'auteur: «On dirait une maladie: je suis atteinte de bilinguisme», déclare Elsa Triolet (citée dans Klein-Lataud 1996: 216). La présence de plusieurs codes linguistiques ne résulte donc pas toujours d'un choix volontaire, contrairement à ce qu'avance Jorge Semprun (cité dans Klein-Lataud 1996: 212).

Les tensions linguistiques engendrées par les deux guerres mondiales et les migrations massives ont fait naître un sentiment d'insécurité linguistique tant chez les auteurs exilés que chez ceux qui demeurent dans leur pays. Les écrivains modernes (et postmodernes) finissent par ne plus se sentir «chez eux» dans leur langue (Steiner 1992: 185), ce qui les amène à se battre tant bien que mal contre elle: «Established language is the enemy» (Steiner 1992: 186). Dans un tel contexte, il n'est pas étonnant que certains écrivains choisissent de rédiger une partie de leur œuvre dans une langue étrangère. Par exemple, Forster explique que le français est un véritable exutoire pour 
Rainer Maria Rilke, car "[French] words are not burdened with irrelevant associations» (Forster 1970: 66).

Mais qu'arrive-t-il lorsque les auteurs modernes ont l'impression qu'ils «manquent » de mots et qu'aucune langue n'est apte à communiquer leurs émotions? Selon Steiner, ils cherchent à renouveler leur langage pour échapper au silence, utilisant trois méthodes: "a process of dislocation, an amalgam of existing languages, or a search for self-consistent neologism» (Steiner 1992: 196). En enfreignant les règles grammaticales de leur langue maternelle ou en y insérant des mots étrangers et des trouvailles personnelles, les poètes modernes ont donc fait renaître le prestige de la littérature polyglotte: "Le bilinguisme littéraire, plutôt que d'être une entrave à la production, est reconnu comme source d'innovation et d'interférence créatrice» (Simon 1996: 56). Si l'on en croit Steiner, les créations collectives multilingues des poètes de l'avant-garde représentent probablement l'exemple extrême de l'expérimentation multilingue en littérature moderne (Steiner 1992: 198).

Depuis quelques dizaines d'années, l'hétéroglossie littéraire est présente à un tel point qu'elle «hante l'écriture postmoderne» (Simon 1996: 55). À l'ère de la mondialisation, les populations se mélangent, les frontières s'effacent et les littératures se diversifient au rythme des migrations. Les «sociétés plurilingues» ne font plus exception; on pourrait même argumenter qu'elles sont devenues la norme (Mackey 1993: 48; Simon 1994: 183). Plusieurs communautés multilingues construisent leur identité en produisant une littérature résolument hétérolingue (Lewis 2003: 412). Simon applaudit l'originalité et l'ingéniosité de ces littératures émergentes: «En interrogeant les imaginaires de l'appartenance, en faisant état de dissonances et d'interférences textuelles, le texte plurilingue fait état des bouleversements socio-démographiques et conceptuels de notre époque, et fait appel à de nouveaux modes de symbolisation » (Simon 1996: 55-56). À la naissance du XXI ${ }^{\mathrm{e}}$ siècle, les littératures «nationales» reflètent les sociétés plurilingues dont elles sont issues.

Ce bref survol de l'histoire littéraire démontre à quel point le multilinguisme est omniprésent dans la littérature. Tantôt une mode, tantôt une exception, la pluralité des langues semble avoir existé un peu partout à presque toutes les époques. D'ailleurs, la grande quantité d'études qui se penchent sur le phénomène tendent à confirmer son importance. Dans la section suivante, nous aborderons les différentes façons dont les théoriciens définissent le multilinguisme, ainsi que les diverses manifestations et fonctions que ce dernier peut avoir en littérature.

\section{Le multilinguisme littéraire dans tous ses états}

\subsection{Manifestations}

András Horn définit trois contextes de cohabitation de diverses langues en littérature ayant apparu simultanément ou séparément à travers l'histoire: 1) dans différentes œuvres au sein d'une même littérature nationale; 2) dans différentes œuvres d'un même auteur; 3) dans une même œuvre présentant un mélange de plusieurs langues (Horn 1981: 225). Peu importe s'ils qualifient cette littérature de «bilingue» (Sarkonak et Hodgson 1993: 13), de «multilingue» (Grutman 2004: 157) ou de "plurilingue» (Mackey 1993: 46; Elwert 1960: 410), les théoriciens se réfèrent presque toujours aux textes comprenant ce que Lewis appelle «interpénétration horizontale» 
des langues (Lewis 2003: 411), synonyme inédit de l'expression plus courante «alternance de codes» (Kürtösi 1993: 107). Ainsi, la plupart des critiques préfèrent restreindre leurs analyses à la troisième situation décrite par Horn, soit les textes où apparaissent conjointement plusieurs idiomes (Elwert 1960: 410; Giese 1961: 80; Grutman 2004: 157; Kürtösi 1993: 107; Sarkonak et Hodgson 1993: 17).

En général, on s'attend à ce que l'auteur d'un texte multilingue y «mélange» sa langue «maternelle» avec une langue "étrangère». Néanmoins, les idiomes utilisés ne sont pas forcément si éloignés l'un de l'autre. En effet, les écrivains issus de sociétés bilingues peuvent entremêler deux ou plusieurs langues qui sont toutes considérées "maternelles» au sein de leur communauté linguistique, comme l'illustrent les textes des auteurs chicanos contemporains qui combinent souvent l'anglais et l'espagnol (Grutman 2004: 157). En outre, il appert qu'un texte peut également être considéré comme polyglotte lorsqu'on y relève l'usage de dialectes, de sociolectes, de néologismes personnels d'un auteur ou la présence d'une langue «artificielle» ou «inventée» (Grutman 2004: 158; Elwert 1960: 412-416). Vu sous cet angle, le multilinguisme englobe une très grande quantité d'œuvres de tous genres.

Le nombre de cas grimpe encore si l'on considère que la quantité d'éléments hétérogènes apparaissant dans le texte peut varier d'un seul mot à des passages entiers (Elwert 1960: 415; Grutman 2004: 158; Kürtösi 1993: 107). Elwert ajoute que la fréquence des insertions change: l'œuvre au complet ou seulement certaines parties isolées peuvent être affectées, et cela «d'une manière régulière [...] [ou] selon un procédé systématique» (Elwert 1960: 415). Schogt signale que ces incursions peuvent être plus ou moins «intégrées» au texte «by orthographic and/or morphological adaptation to the systems of the receiving language» (Schogt 1988: 112). Sarkonak et Hodgson mentionnent eux aussi le caractère plus ou moins flagrant de l'" étrangeté", "ranging all the way from outright code-switching - where italics would normally be used to underscore the change in code - to more subtle cases of linguistic 'interference' » (Sarkonak et Hodgson 1993: 16).

Sternberg situe les textes multilingues entre deux pôles: vehicular matching, où le discours hétérolingue des personnages est reproduit dans sa version "originale", et homogenizing convention, où l'auteur normalise les citations en les rapportant carrément dans la langue principale de la narration (Sternberg 1981 : 223-224). Ainsi, le plurilinguisme se situerait davantage du côté du vehicular matching et compterait deux manifestations principales: la "reproduction sélective» et la «transposition verbale» (Sternberg 1981 : 225). D’abord, la «reproduction sélective» se caractérise par l'apparition sporadique des paroles d'un personnage dans la «langue originale», parfois accompagnées d'une traduction entre parenthèses pour faciliter la compréhension des lecteurs ${ }^{2}$ (Sternberg 1981: 226-227). Ensuite, la "transposition verbale» concerne les cas d'interférence, où la langue principale de l'ouvrage reflète certaines caractéristiques (lexicales, morphologiques ou syntaxiques) de la langue «étrangère » (Sternberg 1981 : 227-228). À ce sujet, Lewis donne l'exemple des littératures postcoloniales, où plusieurs auteurs "ont essayé de faire entendre leur voix à travers des langues européennes qui leur avaient été imposées ${ }^{3}$. [...] En passant leur texte par la matrice de leurs propres cultures [...], ces écrivains finissent par tisser un texte dont le contenu [...] est le produit de plus d'une culture, plus d'une langue ou plus d'une expérience du monde» (Lewis 2003: 416). 


\subsection{Fonctions}

Un auteur peut avoir plusieurs raisons d'intégrer des éléments linguistiques étrangers à son texte. Par exemple, de nombreux théoriciens croient que les auteurs ont avant tout recours à des langues étrangères pour brosser un portrait plus réaliste d'une société/communauté bilingue ou pour souligner la nationalité d'un personnage (Elwert 1960: 417; Giese 1961: 83; Sarkonak and Hodgson 1993: 24; Schogt 1988: 112). En outre, un auteur peut chercher à donner une certaine «élégance cosmopolite» au texte (Giese 1961: 87; Grutman 1993: 224; Schogt 1988: 113) ou même à «étaler ses connaissances linguistiques» (Elwert 1960: 417). À moins qu'il n'ait tout simplement envie d'explorer les sonorités étrangères pour son propre plaisir (Sarkonak and Hodgson 1993: 24). Horn a étudié la situation plus en détail et établi une liste exhaustive qui résume bien les principaux rôles de l'hétéroglossie littéraire:

1. sprachlich charackterisieren [caractériser (un personnage)]

2. die Illusion fördern [accentuer l'illusion (de réalité)]

3. Träger auktorialer Aussage sein [établir le ton de l'auteur]

4. zur Einheit in der Mannigfaltigkeit beitragen [contribuer à unir des éléments hétérogènes $\left.{ }^{4}\right]$

5. komische Wirkung haben [produire un effet comique]

6. sprachspezifische Bedeutung vermitteln [transmettre un concept spécifique à une langue $\left.{ }^{5}\right]$

7. ein Faktor lautlicher Schönheit sein [être un facteur purement esthétique]

8. als Zitat fungieren [fonctionner comme citation ${ }^{6}$ ] (Horn 1981 : 226)

Dépendamment du genre littéraire où le multilinguisme se manifeste, l'une ou l'autre des fonctions sera privilégiée. Par exemple, bien que Horn n'en fasse pas explicitement mention, les fonctions numéros 1,2 et 8 laissent penser qu'elles pourraient se restreindre à la prose (fiction ou théâtre), alors que les autres pourraient englober la poésie.

Certes, les problèmes de traduction ainsi que les stratégies pour les résoudre vont dépendre des fonctions et des formes du multilinguisme dans l'original. Or, si le plurilinguisme littéraire semble toujours remplir une fonction quelconque, il est parfois un élément absolument essentiel sans lequel le texte perdrait de la valeur: «an integral part of the text's overall significance" (Sarkonak et Hodgson 1993: 17). Grutman renchérit en avançant qu'un texte ne peut être considéré comme réellement «bilingue» que lorsque le mélange des langues y joue un rôle «pertinent » (Grutman 1993: 209).

En somme, cette synthèse des manifestations et des fonctions du multilinguisme en littérature paraît suggérer qu'un très grand nombre d'œuvres littéraires, tous genres confondus, peuvent être jugées «multilingues» à un degré plus ou moins prononcé. Or, si l'insertion de langues étrangères est si fréquente et utile aux écrivains et qu'elle est souvent tissée à même la trame, il serait souhaitable d'en conserver ne serait-ce que la trace lors de sa traduction. Voyons à présent dans quelle mesure il est possible, selon les théoriciens, de traduire un texte multilingue. 


\section{Le multilinguisme littéraire en traduction}

\subsection{Un silence théorique}

Dans un article paru en 1985, Derrida déplore que les théories de la traduction ne se penchent pas plus souvent sur les œuvres multilingues, qui soulèvent «la possibilité pour des langues d'être impliquées à plus de deux dans le texte ${ }^{7}$ (Derrida 1985: 215). À la suite de l'émergence des études post-coloniales, il est vrai qu'un nombre grandissant de théoriciens s'intéressent à présent aux textes multilingues. Pensons notamment à des ouvrages collectifs tels que Post-Colonial Translation, Changing the Terms et Rethinking Translation ${ }^{8}$. Néanmoins, ces anthologies traitent avant tout d'une "poétique de la traduction": les auteurs y établissent un parallèle convaincant entre l'acte traductif et l'écriture post-coloniale, mais ne font qu'effleurer la possibilité que les textes à l'étude (par ailleurs souvent plus multiculturels que multilingues) soient eux-mêmes traduits dans une autre langue. Il appert donc que Lewis a raison lorsqu'il déclare que les théoriciens ne se penchent toujours pas assez concrètement sur les «défis que posent ces variétés [hybrides] à l'égard des conceptions conventionnelles de la langue et de la traduction» (Lewis 2003: 412). En fait, on dirait que les traductologues ne se sont pas encore vraiment attaqués aux problèmes que peut engendrer la traduction d'un texte multilingue.

D’abord, plusieurs ne concèdent que quelques lignes au multilinguisme et baissent souvent les bras avant même d'avoir exploré le sujet. Par exemple, Steiner promet de revenir plus loin sur les problèmes de traduction que soulève l'hybridité linguistique dans un passage traitant d'une création collective multilingue moderne (Steiner 1992: 198). Néanmoins, force est de constater à la lecture de son livre que Steiner ne tient pas promesse. De même, Sternberg se contente de soulever la question à la toute fin de son article: "what happens to a translational mimesis in translation, especially when the new target language is none other than the heterolingual source imitated by the original text?» (Sternberg 1981 : 239). Non seulement ne donne-t-il pas l'ombre d'une réponse, mais il souligne également qu'il ne veut pas du tout participer au débat: " $M y$ purpose has been to demonstrate the power and range of translation [i.e. polyglot writing], not to legitimate the vagaries of translators» (Sternberg 1981:239).

\subsection{Une voie sans issue?}

La plupart de ceux qui tentent de répondre à la question sont habités d'un pessimisme écrasant. La réponse de Derrida est plutôt déprimante. En effet, le critique se contente de constater avec fatalité l'impossibilité de traduire l'hybridité linguistique: «la traduction peut tout, sauf marquer [...] cette différence de système de langues inscrite dans une seule langue; à la limite elle peut tout faire passer [...] sauf le fait qu'il y a, dans un système linguistique, peut-être plusieurs langues»(Derrida 1982: 134). Même Berman, normalement assez optimiste quant à la possibilité de relever «l'épreuve de l'étranger», baisse les bras devant la «menace» que la traduction fait planer sur «la superposition des langues ». Il conclut que l'hétérogénéité linguistique «tend à s'effacer» dans la plupart des traductions qu'il connaît (Berman 1985: 79) et ne voit pas comment se sortir de l'impasse.

Un des seuls qui s'attarde vraiment sur le sujet est Schogt, dans le $17^{\mathrm{e}}$ chapitre de son livre intitulé Linguistics, Literary Analysis, and Literary Translation (1988). À 
première vue, Schogt semble vouloir esquisser une ébauche de «théorie» et débute en disant que "as a rule only the main language of the text is replaced, the foreign elements remaining unchanged» (Schogt 1988: 114). Par contre, la "règle» perd du poids lorsque les langues imbriquées de l'original appartiennent à la même famille et sont «compréhensibles» par les locuteurs de la langue principale. Dans ces cas-là, dit Schogt, le traducteur fera souvent fi des différences linguistiques et traduira tout d'un bloc (Schogt 1988: 114). Ensuite, les complications se multiplient. D'abord, Schogt reconnaît qu'un élément «étranger» n'a pas la même connotation pour toutes les cultures: "ethnic stereotypes and prejudices vary from one people to another" (Schogt 1988: 114). Il en conclut que les solutions miracles n'existent pas: "one has to be resigned to accept this state of affairs» (Schogt 1988: 114). Il ne trouve pas non plus d'issue au problème épineux qui surgit lorsque la langue de la traduction est la même que celle des éléments hétérogènes de l'original (Schogt 1988: 114). Mais il y a plus: au tout début de son texte, Schogt écrivait que l'importance de la fonction de l'élément étranger déterminerait si on doit ou non le reproduire dans la traduction (Schogt 1988: 112). Pourtant, il introduit un bémol à cette "règle» à la fin du chapitre: "the literary importance of an element is far from being a guarantee that the translation will maintain it» (Schogt 1988: 119). Au bout du compte, Schogt croit qu'aucune théorie ne peut aider convenablement le traducteur, dont les choix sont de nature purement "pratique» (Schogt 1988: 119). Au terme de la lecture de cet article, il semble impossible de dresser des règles universelles de la traduction des textes multilingues. Au contraire, les traducteurs semblent condamnés à traiter les problèmes cas par cas.

\subsection{La sourde oreille}

En vérité, on dirait bien que la «règle» de traduction s'approche plus de la «négation» pure et simple du «métissage linguistique» que de sa conservation (Lewis 2003: 418). Du moins, plusieurs théoriciens semblent enclins à le croire. Notamment Heiss, qui remarque dans son étude récente sur le doublage des films multilingues: «Examples of flattening out and the formalisation of authentic-sounding spoken language are to be found in almost all dubbed versions, whatever the genre» (Heiss 2004: 213). Dans le même sens, Patry fait l'observation suivante lors d'une analyse de la traduction du vocabulaire anglais francisé de Jacques Ferron: «l'intégration complète à la langue cible est la stratégie [...] qui a été de très loin privilégiée dans l'ensemble des traductions et pour l'ensemble des traducteurs» (Patry 2001: 457). Aussi, Mezei remarque que plusieurs des traducteurs anglais de textes rédigés en français québécois ignorent les incursions anglaises dans le texte français (Mezei 1988: 13).

Toutefois, tous conviennent que le fait d'enrayer complètement l'hétérogénéité du code original peut avoir des effets indésirables. Selon Patry, un «nivellement» des langues «déforme» le texte sur les plans culturel, stylistique et idéologique: il «fait taire [...] la voix de l'étranger», en "gomme le relief» langagier et risque même d'en altérer le contenu en «le rapprochant des valeurs dominantes d'une société donnée» (Patry 2001: 456). Par exemple, une traduction en anglais standard d'un français québécois volontairement truffé d'anglicismes expose le texte à perdre son identité: "[it] can give us a biased and modified impression of Quebec. Quebec becomes not what it is, but what we wish it to be» (Mezei 1988: 18). En outre, la qualité de l'œuvre 
et même sa cohérence interne peuvent se trouver gravement affectées par une uniformisation systématique de son langage. Heiss donne notamment l'exemple du film multilingue Le Mépris de Jean-Luc Godard, où le personnage d'interprète est central. Or, dans les versions doublées en un idiome uniforme et standard, l'interprète devient non seulement superflu, mais carrément ridicule: "the film audience [of the dubbed versions] was presented with a person, who spent part of her time repeating words in a meaningless fashion and partly reciting senseless, newly invented chunks of dialogue " (Heiss 2004 : 208). Patry a bien raison de déclarer que lorsqu'il s'agit de textes jouant avec différentes langues, « une traduction juste du contenu langagier n'est pas nécessairement garante d'une bonne traduction littéraire» (Patry 2001: 456).

\subsection{La vulgarisation pour grand public}

Pourquoi la pratique homogénéisante continue-t-elle d'être si populaire malgré les conséquences fâcheuses qu'elle entraîne? Certains attribuent cette situation à des préoccupations d'ordre financier. En effet, on dirait que les traducteurs (probablement sous l'influence des maisons d'édition) orientent souvent leurs efforts vers la création d'un texte homogène, parce que ce dernier sera "plus facile à comprendre » et "plus agréable à lire» (Mezei 1988: 20). Ainsi, le fait de vouloir (ou de devoir) produire un texte qui se vendra bien peut inciter certains traducteurs à «l'homogénisation» du code linguistique. Selon Heiss, ce phénomène serait particulièrement fréquent (et flagrant) dans le domaine du doublage de films, où règne «the widespread practice of trying to meet the presumed expectations of the target audience» (Heiss 2004: 213).

Les traducteurs qui uniformisent le texte plurilingue tiennent probablement pour acquis que le public de l'original comprenait toutes les langues qui y étaient imbriquées. Or, l'hétéroglossie d'un texte crée une certaine tension indépendamment des qualifications linguistiques des lecteurs visés (Sternberg 1981: 222). En fait, rien n'indique que le texte de départ s'adressait à des lecteurs bilingues ou polyglottes (Grutman 2004: 158). Un auteur peut même délibérément insérer des éléments dans une langue qu'il sait inconnue à son public. Selon Elwert, ce recours «entraîne deux conséquences: 1) l'élément étranger est très réduit, limité à quelques mots ou à un bout de phrase, et 2) il doit être immédiatement suivi de la traduction ou d'une explication» (Elwert 1960: 422). La lecture d'un tel texte ne requiert donc normalement qu'«un peu d'imagination» (Grutman 2004: 158). Finalement, le plurilinguisme littéraire deviendrait problématique lorsque sa compréhension exigerait réellement la connaissance d'une autre langue (Grutman 1993: 212). Peu importe le degré d'hétérogénéité linguistique du texte, une chose est sûre: le multilinguisme du discours encourage le lecteur à participer activement au processus de son décodage (Sarkonak et Hodgson 1993: 9-10). Pour cette raison, un traducteur qui choisirait d'uniformiser le code linguistique de l'œuvre aurait plutôt l'air de donner du tout cuit à ses lecteurs, sous-estimant d'une part leurs capacités d'interprétation et les incitant d'autre part à la passivité.

Ceci étant dit, on ne peut nier l'importance pour le traducteur de bien considérer le contexte de réception de sa version s'il veut que sa traduction soit lue et comprise au même titre que l'original l'était par son public: "Dans cette optique, il incombe au traducteur de connaître le contexte de réception du texte afin d'être capable de modifier son comportement conformément à ce contexte» (Lewis 2003: 
419). Après tout, le but n'est pas de produire un chaos incompréhensible, mais de générer un organisme complexe que les lecteurs pourront apprivoiser : «le traducteur [...] cherche [...] à créer, selon les outils linguistiques d'une communauté réceptrice, des textes significatifs» (Lewis 2003: 419).

Chose certaine, qu'il soit ou non en mesure de conserver l'hétérogénéité linguistique de l'œuvre, le traducteur d'un texte polyglotte a besoin vraisemblablement de plus que de simples connaissances linguistiques pour remplir sa dure mission avec succès. En fait, tous les facteurs suivants devraient entrer en interaction dans le processus traductif: "the particular system of the text, the system of the culture out of which the text has sprung, and the cultural system in which the metatext will be created» (Mezei 1988: 15). Pour être capable de juger quand et où il est possible ou nécessaire de reproduire les éléments hétérogènes, le traducteur devra non seulement bien connaître la culture du public cible, mais aussi comprendre celle du public source. Aussi devra-t-il se familiariser avec le texte et être sensible aux mécanismes qui le sous-tendent.

\subsection{Le culte de la norme}

Dans un autre ordre d'idées, il est possible que les raisons d'une standardisation massive des codes multiples soient ancrées beaucoup plus profondément que dans les exigences mercantiles. En fait, elles pourraient s'enraciner à même l'éthique de la traductologie, qui tend à privilégier une conception «prescriptive» du langage. Cela concerne tant l'enseignement que la pratique de la traduction, où l'on cherche généralement à rédiger des textes grammaticalement corrects et idiomatiques. Une telle orientation fait en sorte que les traducteurs conçoivent souvent «[...] toute manifestation d'hétérogénéité linguistique [...] comme une aberration» (Lewis 2003: 412). Par exemple, bien que Steiner avoue que l'acte traductif puisse viser à « réinventer la forme de la signification ", il considère néanmoins qu'il comporte toujours une tendance à «abolir la multiplicité» (Steiner 1992: 246). La rubrique "Multilingualism and Translation", dans la Routledge Encyclopedia of Translation Studies, va plus loin en établissant une dichotomie claire entre multilinguisme et traduction: "multilingualism evokes the co-presence of two or more languages (in a given society, text or individual), translation involves a substitution of one language for another " (Grutman 2004: 157). Selon cette définition, la traduction serait donc inapte à rendre un texte multilingue, puisque le processus traductif implique systématiquement un remplacement qui en effacerait la multiplicité. Ainsi, comme la littérature multilingue n'est pas rédigée dans un idiome officiel mais dans une «langue métissée» qui fait briller les contrastes, il n'est pas étonnant qu'elle fasse naître chez plusieurs traducteurs un désir de «standardiser» le discours hétérogène.

Il va sans dire que le traducteur devra faire preuve d'une grande ouverture à l'Autre s'il veut que sa traduction soit elle aussi «ouverte»: "open to differences, open to varieties of meanings, open to both the original cultural referential system and the one in the process of being created" (Mezei 1988: 20). Pour ce faire, le traducteur ne doit pas craindre de jouer avec sa langue, de laisser l'Autre y pénétrer jusqu'à en altérer la structure. Même si une traduction polyglotte n'aura jamais un effet tout à fait identique à celui de l'original, elle aura le mérite d'avoir reconnu sa nature hétérogène et d'avoir osé la reproduire, ce qui est en soi déjà beaucoup mieux que d'ignorer 
totalement le phénomène. À propos des effets bénéfiques dans le domaine du doublage de films, Heiss conclut que "the creative use of these means [compensation at syntactic, lexical, pragmatic and phonetic levels] could produce dubbed versions that are much more satisfying and appropriate than those containing the smoothed over and levelled out 'dubbese' so often presented in the so-called dubbing countries" (Heiss 2004: 211). Dans certains cas, la traduction peut même être plus étrange que l'original, sans être nécessairement un mal pour autant.

\subsection{Une lueur d'espoir}

En fait, ce ne sont souvent pas les théoriciens de la traduction, mais plutôt les traducteurs eux-mêmes qui sont les mieux placés pour parler des solutions concrètes aux problèmes que pose le multilinguisme littéraire. Peut-être justement parce qu'ils sont plus pratiques que théoriques, deux récents articles fournissent notamment des pistes intéressantes: «Life Is a Caravanserai: Translating Translated Marginality, a Turkish-German Zwittertext in English», de Luise von Flotow et «Stratégies de traduction et non-traduction dans The Widows de Suzette Mayr», de Nathalie Ramière?. Larticle de von Flotow traite de sa traduction anglaise d'un roman rédigé en un étrange allemand truffé d'interférences turques, tant sur les plans formel (lexical, grammatical, morphologique) que thématique. Quant à elle, Ramière écrit également sur sa propre traduction d'un roman albertain racontant l'histoire de trois immigrantes allemandes au Canada, où l'hybridité culturelle se reflète dans la langue de la narration.

Dans les deux cas, les traductrices jugent que le multilinguisme est une caractéristique intrinsèque tant du texte original que de sa traduction. Sans contredit, le fait que chacun des deux romans revête les huit fonctions de l'hétéroglossie littéraire répertoriées plus haut par Horn leur donne raison (voir point 2.2 du présent article). D’abord, l'hétérogénéité de la langue sert à caractériser de façon réaliste respectivement la communauté turque en Allemagne et la communauté allemande dans l'Ouest canadien [1], accentuant ainsi l'illusion de réalité de la narration [2]. D’ailleurs, cela se fait souvent sous forme de citation dans les deux œuvres [8]. Ensuite, l'hybridité linguistique établit le ton très personnel et original de l'auteure [3]. En outre, la langue contribue à unir les cultures représentées (allemande-turque, allemande-canadienne), généralement très éloignées l'une de l'autre [4]. Enfin, le mélange linguistique sert souvent à transposer dans la langue de la narration des concepts spécifiquement étrangers [6], générant parfois au passage un effet comique [5] ou encore poétique [7]. En conséquence, il n'est pas étonnant que tant von Flotow que Ramière aient tenté de conserver l'hybridité linguistique du texte original dans leur traduction.

Puisque le multilinguisme de Life is a Caravanserai est souvent lui-même issu de calques turques en allemand, von Flotow a opté dans la plupart des cas pour un calque du calque, soit une traduction en anglais "aussi littérale que possible» des traductions littérales du turque en allemand (von Flotow 2000: 68). Entres autres, les structures grammaticales qui sont hétéroclites en allemand le sont également en anglais, comme par exemple l'absence délibérée de complément d'objet direct là où ce dernier serait grammaticalement nécessaire (von Flotow 2000: 70). Aussi, les emprunts directs à l'arabe ou au turque sont reproduits tels quels en anglais (von Flotow 2000 : 71). Outre le fait que ces procédés soulignent l'appartenance de l'œuvre 
à une littérature minoritaire, von Flotow ajoute que le fait de calquer le vocabulaire et les structures boiteuses de l'original «may even allow English readers/speakers of Turkish to understand and enjoy some of the subtext of hidden wordplay» (von Flotow 2000 : 68). Selon von Flotow, son approche traductive «littérale» réussit à produire un mélange linguistique similaire qui non seulement transmet, mais accentue la «différence» qui caractérisait l'original (von Flotow 2000: 71)

De façon similaire à von Flotow, Ramière conserve elle aussi le plus d'emprunts directs de l'allemand que possible dans sa traduction française, afin de «marquer [son] texte comme "étranger" et de conserver la dimension humoristique» de l'original (Ramière 2003: 188). En ce qui concerne ce qu'elle nomme les «cross-references » entre l'anglais et l'allemand présentes dans l'original, Ramière a décidé de les garder le plus souvent possible. La présence des références à l'anglais dans le texte français rend la traduction par moments encore plus hybride et métalinguistique que l'original: «Une telle stratégie rappelle évidemment au lecteur qu'il est en train de lire une traduction, mais elle confirme également que la littérature d'immigration est bien “un lieu d'énonciation périphérique" (Simon 1994: 25)»(Ramière 2003: 189). Toutefois, Ramière hésite plus que von Flotow à dépayser les lecteurs de sa traduction. Ainsi, elle a préféré paraphraser les expressions allemandes qu'elle juge incompréhensible à un public francophone. Par exemple, elle traduit «Kaffeeklatsch» par « un truc de commères habituées à prendre le café ensemble» et «Hausfrau» par «maîtresse de maison allemande» (Ramière 2003: 188). Même si nous partageons l'aversion de Ramière pour la note de bas de page, force est de constater que ses paraphrases sont un peu trop lourdes pour être insérées à même le texte. Par ailleurs, on peut se demander si un lecteur francophone est vraiment plus inapte qu'un anglophone à comprendre ces expressions dans le contexte assez clair où elles apparaissent. En fait, l'article de Ramière semble confirmer que le principal obstacle auquel se heurte le traducteur de textes multilingues n'est pas nécessairement linguistique, mais surtout psychologique.

Malgré le fait que l'attitude de Ramière semble un peu moins radicale que celle de von Flotow, il est intéressant de constater que les deux traductrices utilisent des procédés de traduction similaires. En effet, toutes deux optent pour la conservation d'emprunts directs ainsi que pour la littéralité comme solutions traductives aux défis posés par le multilinguisme du texte original. Au terme de la lecture de ces articles, il est clair qu'il est non seulement souhaitable, mais possible, de traduire l'hybridité linguistique d'un texte. Dans certains cas, il s'agit tout simplement d'oser calquer l'original.

\section{Synthèse et éléments de conclusion}

En définitive, la présente étude souligne l'importance pour les traducteurs et traductologues de développer de nouvelles stratégies capables de reproduire adéquatement le multilinguisme littéraire. D’abord, notre bref survol historique démontre que l'hétéroglossie n'est pas née d'hier et qu'elle est même loin de vouloir disparaître. Aussi, les multiples efforts des théoriciens littéraires pour déterminer les fonctions du phénomène et ses diverses manifestations tendent à confirmer son importance. Si les traducteurs ont toujours été aux prises avec les difficultés inhérentes à la traduction d'éléments linguistiques hétérogènes, ils ne peuvent plus faire la sourde 
oreille aujourd'hui, à une époque où, comme le dit si bien Wuilmart, on assiste à une «volonté croissante de respecter la culture du voisin, en dépit des difficultés techniques et pratiques parfois insurmontables» (Wuilmart 1994: 251). D'ailleurs, le fait de remplacer un code hétérogène par un autre plus «standard» risque d'entraîner des conséquences néfastes sur tous les plans. En plus de mettre en péril la cohérence et d'altérer l'esthétique du texte, l'uniformisation du multilinguisme peut avoir aujourd'hui de graves implications culturelles ou même politiques, surtout lorsqu'il s'agit d'une littérature dite «minoritaire». Il semblerait donc que ce n'est pas tant la nature des textes multilingues modernes qui justifie une traduction multilingue, mais bien plutôt leurs contextes de production et de réception.

Certes, les textes plurilingues présentent une confusion linguistique digne de Babel qui en complexifie la traduction. Néanmoins, ce défi ne signifie pas pour autant qu'ils sont absolument intraduisibles. En fait, l'impossibilité de traduire est démentie par l'industrie même de la traduction. Comme l'explique Steiner: "We do translate intra- and interlingually and have done so since the beginning of human history» (Steiner 1992: 264). Cette affirmation inclut aussi les textes polyglottes. Après tout, même le Finnegans Wake de Joyce, sempiternel exemple d'intraduisibilité que les théoriciens (dont Derrida) se plaisent tant à citer, est paru en diverses langues ${ }^{10}$. Sans connaître la «qualité» de ces versions, leur seule existence suggère qu'il est au moins envisageable d'arriver à des résultats "publiables». De nos jours, selon de Pedro, il faut reconnaître le fait que «absolute untranslatability, whether linguistic or cultural, does not exist [anymore]»(De Pedro 1999: 556). Ceci dit, les traducteurs sont conscients que la transposition d'un texte d'une langue à une autre engendre forcément des «pertes». L'inaccessible n'est pas la traduction comme telle, mais plutôt sa «perfection» (De Pedro 1999: 556).

En fait, il ressort que les textes multilingues ne remettent pas en question la notion de traductibilité, mais plutôt celle de traduction elle-même. Ainsi, Simon avance que les textes polyglottes, parce qu'ils ont eux-mêmes un caractère «incomplet», exigent une traduction portant les traces de cette incomplétude et ne menant «pas toujours à un résultat homogène mais qui - à l'instar des identités culturelles du monde contemporain - se confronte en permanence à l'inachevé» (Simon 1994: 181). Un texte multilingue est non seulement «étranger», mais aussi «étrange», et sa traduction devrait être à son image. Cela semble impliquer que les textes multilingues exigeraient une traduction totalement «dépaysante» (foreignizing) où l'hétérogénéité de l'original est encore perceptible. Or, Berman invitait déjà les traducteurs littéraires à «accueillir l'Autre» dans sa populaire étude L'Épreuve de l'étranger. En réponse à Derrida (1985: 215), nous proposons donc qu'une traduction en «plusieurs langues à la fois» demeure de plein droit une traduction, mais dans un sens plus large que celui auquel on nous a habitués, un sens plus englobant, plus représentatif des hybridités grandissantes du monde d'aujourd'hui.

\section{NOTES}

1. Malgré tout, certains auteurs romantiques, fascinés par les cultures des pays lointains, insèrent des éléments linguistiques «exotiques» dans leurs textes: «Sobre todo el exotista Pierre Loti ofrece bellos ejemplos de elementos de lenguas orientales para caracterizar el ambiente» [En particulier l'exotiste Pierre Loti offre de beaux exemples d'éléments en langues orientales pour caractériser l'ambiance] (Giese 1961: 81). 
2. La méthode de «traduction» que décrit Grutman va un peu dans le même sens, puisqu'elle consiste à citer les paroles du personnage directement, tout en incluant systématiquement une traduction des passages étrangers, ce qui permet au lecteur de «sauter» ce qu'il ne comprend pas (Grutman 1993: 210).

3. Cependant, une langue étrangère n'a pas besoin d'avoir été «imposée» pour être piquée d'interférences de la langue maternelle. Notamment, L'Écriture ou la vie compte quelques passages où le français de Semprun paraît calqué sur la grammaire espagnole. L'extrait suivant, où il y a élision systématique des sujets, est particulièrement frappant: "Ils sont sortis de la voiture à l'instant, il y a un instant. Ont fait quelques pas au soleil, dégourdissant les jambes. M'ont aperçu alors, se sont avancés» (Semprun 1994: 14).

4. Horn donne l'exemple du «refrain» (Horn $1981: 233$ ).

5. Ici, Horn mentionne que le poète hongrois Miklös Radnóti a préféré le terme Lager (camp de concentration) à son équivalent dans sa langue maternelle, car le mot allemand est plus chargé symboliquement (Horn 1981: 238). Dans la même veine, on pense à la phrase «Krematorium ausmachen!» [Éteignez le crématoire!], récurrente en allemand dans L'écriture ou la vie de Jorge Semprun.

6. Horn fait écho à Schogt et Grutman en précisant que les citations empruntées à des langues «prestigieuses» «rehaussent» le niveau du texte et lui donnent une certaine élégance (Horn 1981: 239).

7. Un lecteur averti serait probablement tenté de demander à Derrida pourquoi il n'aborde lui-même la question que du bout des lèvres.

8. Post-Colonial Translation. Theory and Practice (1999): Ed. by Susan Bassnett and Harish Trivedi, London and New York, Routledge; Changing the Terms: Translating in the Post-Colonial Era (2000): Ed. by Sherry Simon and Paul St-Pierre, Ottawa, University of Ottawa Press; Rethinking Translation: Discourse, Subjectivity, Ideology (1992): Ed. by Lawrence Venuti, London and New York, Routledge.

9. Luise von Flotow (2000): «Life Is a Caravanserai: Translating Translated Marginality, a TurkishGerman Zwittertext in English», Meta, 45-1, pp. 65-72; Nathalie Ramière (2003): "Stratégies de traduction et non-traduction dans The Widows de Suzette Mayr», TTR, 16-2, pp. 175-196.

10. Un coup d'œil sur l'Index Translationum de l'Unesco suffit: une dizaine de traductions y sont mentionnées, notamment en français, allemand, espagnol, néerlandais et même une en japonais.

\section{RÉFÉRENCES}

Bassnett, S. and H. Trivedi (1999): Post-Colonial Translation. Theory and Practice, London and New York, Routledge.

Berman, A. (1985) : "La traduction comme épreuve de l'étranger», Texte 4: Traduction: Textualité / Text: Translatability, Toronto, Trinity College, pp. 67-81.

De Pedro, R. (1999): «The Translatability of Texts: A Historical Overview», Meta 44-4, pp. 546559.

DERRIDA, J. (1982) : «L'oreille de l'autre: otobiographies, transferts, traductions », in LÉvESQUE, C. et C. V. Macdonald (dir.), Textes et débats avec Jacques Derrida Montréal, VLB, pp. 125212.

Derrida, J. (1985): «Des Tours de Babel», in Graham, J. F. (ed.), Difference in Translation, Ithaca and London, Cornell University Press, pp. 209-248.

Elwert, W. Th. (1960): "L'emploi de langues étrangères comme procédé stylistique», Revue de littérature comparée 34, pp. 409-437.

Forster, L. (1970): The Poet's Tongues: Multilingualism in Literature, London, New York and Sydney, Cambridge University Press.

Giese, W. (1961) : «El empleo de lenguas extranjeras en la obra literaria», Studia Philologica II, Homenaje ofrecido a Dámaso Alonso por sus amigos y discípulos con ocasión de su $60^{\circ}$ aniversario, Madrid, Editorial Gredos, pp. 79-90.

Grutman, R.(1990): «Le bilinguisme littéraire comme relation intersystémique», Canadian Review of Comparative Literature - Revue canadienne de littérature comparée 17/3-4, pp. 198-212. 
Grutman, R. (1993): «Mono versus Stereo: Bilingualism's Double Face», Visible Language, 27/1-2, pp. 206-226.

Grutman, R. (2004): «Multilingualism and Translation», in Baker, M. (ed.), Routledge Encyclopedia of Translation Studies, London and New York, Routledge, pp. 157-160.

HeIss, C. (2004): «Dubbing Multilingual Films: A New Challenge?», Meta 49-1, pp. 208-220.

Horn, A. (1981): « Ästhetische Funktionen der Sprachmischung in der Literatur », Arcadia 16-3, pp. 225-241.

Klein-Lataud, C. (1996): «Les voix parallèles de Nancy Huston», TTR 9-1, pp. 211-231.

Kürtösı, K. (1993): «Poets of Bifurcated Tongues, or on the Plurilingualism of CanadianHungarian Poets", TTR 6-2, pp. 103-130.

Lewis, R. A. (2003): «Langue métissée et traduction: quelques enjeux théoriques», Meta 48-3, pp. $411-420$.

Mackey, W. (1993): «Literary Diglossia: Biculturalism and Cosmopolitanism in Literature», Visible Language 21/1-2, pp. 40-66.

Mezei, K. (1988): «Speaking White: Literary Translation as a Vehicle of Assimilation in Quebec», Canadian Literature - Littérature canadienne 117, pp. 11-24.

Patry, R. (2001): «La traduction du vocabulaire anglais francisé dans l'œuvre de Jacques Ferron: une impossible épreuve de l'étranger» Meta 46-3, pp. 449-466.

Ramière, N. (2003) : «Stratégies de traduction et non-traduction dans The Widows de Suzette Mayr», TTR 16-2, pp. 175-196.

Sarkonak, R. and R. Hodgson (1993): "Seeing in Depth: The Practice of Bilingual Writing", Visible Language 21/1-2, pp. 6-39.

Schogt, H. G. (1988): «Foreign Languages and Dialects», Linguistics, Literary Analysis and Literary Translation, Toronto, Buffalo and London, University of Toronto Press, pp. 112119.

Semprun, J. (1994): L'écriture ou la vie, Paris, Gallimard.

Simon, S. and P. St-Pierre (eds) (2000): Changing the Terms: Translating in the Post-Colonial Era, Ottawa, University of Ottawa Press.

Simon, S. (1996): «Entre les langues: Between de Christine Brooke-Rose», TTR 9-1, pp. 55-71.

Simon, S. (1994): Le trafic des langues - Traduction et culture dans la littérature québécoise, Montréal, Boréal.

Steiner, G. (1992): After Babel - Aspects of Language and Translation, New York, Oxford University Press.

Sternberg, M. (1981): "Polylingualism as Reality and Translation as Mimesis», Poetics Today 2-4, pp. 221-239.

Venuti, L. (ed.) (1992): Rethinking Translation: Discourse, Subjectivity, Ideology, London and New York, Routledge.

Von Flotow, L. (2000): «Life is a Caravanserai: Translating Translated Marginality, a TurkishGerman Zwittertext in English», Meta 45-1, pp. 65-72.

Wuilmart, F. (1994): «La traduction littéraire: son "européanisation", sa didactique», Meta 39-1, pp. 250-256. 